\title{
Galactic Dynamos
}

\author{
David Moss \\ Mathematics Department, University of Manchester, Manchester \\ $M 13$ 9PL, UK
}

\begin{abstract}
There is a broad agreement between the predictions of galactic dynamo theory and observations; although there are still some unresolved difficulties, the theory appears to be robust. Now attention is turning from generic models to studies of particular features of the largescale magnetic fields, and also to models for specific galaxies. The effects of noncircular flows, for example driven by the interaction of spiral arms and galactic bars with the dynamo, are of current interest.
\end{abstract}

\section{Introduction}

Fundamental issues concerning the nature of the nonlinear quenching operating in galactic dynamos, and whether the large scale ('regular') field can grow to the observed, approximately equipartition, strengths observed are discussed elsewhere in this Proceedings. This review is written assuming that mean field dynamo theory gives an approximate description of the generation and structure of large scale galactic magnetic fields.

The basis for this belief is partly pragmatic (although other arguments are developed elsewhere in this volume): it appears that fields observed in spiral galaxies are usually of even parity with respect to the galactic midplane, and basically axisymmetric, i.e. they have an overall quadrupole-like structure. The only alternatives that have been at all developed to rival dynamo theory to explain galactic magnetic fields are theories in which a pre-existing field is trapped and amplified as the galaxy forms, and subsequently is distorted by the differential rotation. However, the part of any such 'primeval' field that is parallel to the rotation axis will lead to an axisymmetric field component that is of odd parity with respect to the disc midplane (aligned dipole-like topology), and the part perpendicular to this axis will give a nonaxisymmetric field component with even parity ('perpendicular dipole-like'). On the other hand, basic nonlinear galactic dynamo models quite generally produce stable axisymmetric fields that are of even parity with respect to the midplane, as observed. Non-circular motions, modulation by spiral arms, etc, can then be expected to produce secondary nonaxisymmetric structures. Thus, it appears that theories based on the trapping, compression and advection of pre-existing magnetic fields cannot explain the topology of the commonly observed fields, whereas this arises naturally from dynamo theory. This argument is not intended to rule out the possibility that some memory of a pregalactic field can manifest itself in the contemporary fields, but to stress that dynamo action must play an important rôle. 
Thus, it will be assumed that the standard mean field dynamo equation

$$
\frac{\partial \mathbf{B}}{\partial t}=\nabla \times\left(\left(\mathbf{u}_{\mathrm{rot}}+\mathbf{v}\right) \times \mathbf{B}+\alpha \mathbf{B}-\eta \nabla \times \mathbf{B}\right),
$$

is adequate to describe the behaviour of galactic magnetic fields. Here $\mathbf{u}_{\text {rot }}$ is the circular (rotation) velocity, and $\mathbf{v}$ includes all other large-scale velocity fields. $\alpha$ and $\eta$ are the usual mean field turbulent transport coefficients.

\section{Uncertainties}

In spite of the generally optimistic tone of the above remarks, there are substantial uncertainties attached to each of the terms in Eq. (1). In principle, galaxies being to some degree transparent, the rotation law $\Omega(r)$ is observable. However the presence of nonaxisymmetric structure can cause problems in determining $\Omega$, and there are often substantial uncertainties in our knowledge of $\Omega(r)$ at small galactocentric radii. In the specific case of the galaxy M31, Moss et al. (1998) showed how two different interpolations on the same observation data could lead to magnetic field structures that differed in significant detail.

Much larger uncertainties may be associated with the non-circular velocities $\mathbf{v}$, which cannot be determined globally from observations. These may be significant even in grand design spirals, as density wave theory predicts gas streaming as fast as 5 or $10 \mathrm{~km} \mathrm{~s}^{-1}$ associated with the arms (see, e.g., Moss 1998, Elstner et al. 2000).

The turbulent transport coefficients $\alpha$ and $\eta$ are both poorly known at present. In principle they should both be tensors, $\alpha_{i j}(\mathbf{B})$ and $\eta_{i j k}(\mathbf{B})$. However the forms of these tensors are quite uncertain, and so $\alpha$ and $\eta$ are often taken as scalars. The form of the dependence on $\mathbf{B}$ is quite uncertain, and often the naive representation $\alpha \propto\left(1+\mathbf{B}^{2} / B_{\mathrm{eq}}^{2}\right)^{-1}$ is used.

The distribution of gas through the galaxy influences the form of the nonlinear feedback onto $\alpha$ (and perhaps also $\eta$ ) that limits the large-scale fields at finite amplitude through the magnitude of the equipartition field $B_{\text {eq }}$, and a reasonably accurate determination is necessary for detailed modelling. This is not in general available.

\section{Other nonlinearities}

Usually, only the standard alpha-quenching nonlinearity described above is included in models. There is also the interesting possibility that an (inherently nonlinear) Parker instability in the disc generates an alpha effect (Parker 1992, Moss et al. 1998, Hanasz \& Lesch 1998). Moreover, this mechanism would not be subject to the objection raised against the conventional alpha-effect, that it is quenched before large-scale fields can grow to the observed magnitude (see, e.g., Blackman, Brandenburg, Hughes, this Proceedings); indeed the magnitude of the effective alpha term is expected to increase with the large-scale field strength. Presumably, any such dynamo would be limited at finite amplitude by the buoyancy nonlinearity removing field from the disc into the halo.

In the inner regions of barred galaxies, where regular fields can exceed $10 \mu \mathrm{G}$, Lorentz force torques may drive an accretion flow of order $1 M_{\odot} \mathrm{yr}^{-1}$ 
(Beck et al. 1999). This flow may feed back onto the dynamo action, in which case hydrodynamic galaxy models may need to include Lorentz forces (see e.g. Moss et al. 2000). Moss et al. (2000) also demonstrated that a strong enough axisymmetric radial flow (e.g. from accretion) can inhibit dynamo action. Of course, in barred galaxies, the flows are far from axisymmetric.

\section{Barred and irregular galaxies}

Markedly nonaxisymmetric large scale magnetic fields are observed in barred systems (e.g. Beck, this Proceedings), and strong nonaxisymmetric motions are expected to be present (Athanassoula 1992). It is quite inadequate to regard such dynamo systems as perturbations of more familiar dynamos - non-circular velocities of $\mathrm{O}(100) \mathrm{km} \mathrm{s}^{-1}$ will dominate any dynamo action.

Some progress has been made in dynamo modelling of barred galaxies. For example, the weakly barred 'ring' galaxy IC4214 was studied by Moss et al. (1999), using a hydrodynamically determined velocity field (with no shock), that had been calibrated against observations. The resulting (very nonaxisymmetric!) dynamo field, rotating with the pattern speed, is shown in Fig. 1. The trailing spiral arms emanate from near the corotation radius, and this radius can be expected quite generally to leave its signature on nonaxisymmetric field structures (see also Shukurov 1998). Further studies using velocities from hydrodynamic models include von Linden et al. (1998) and Elstner et al. (2000).

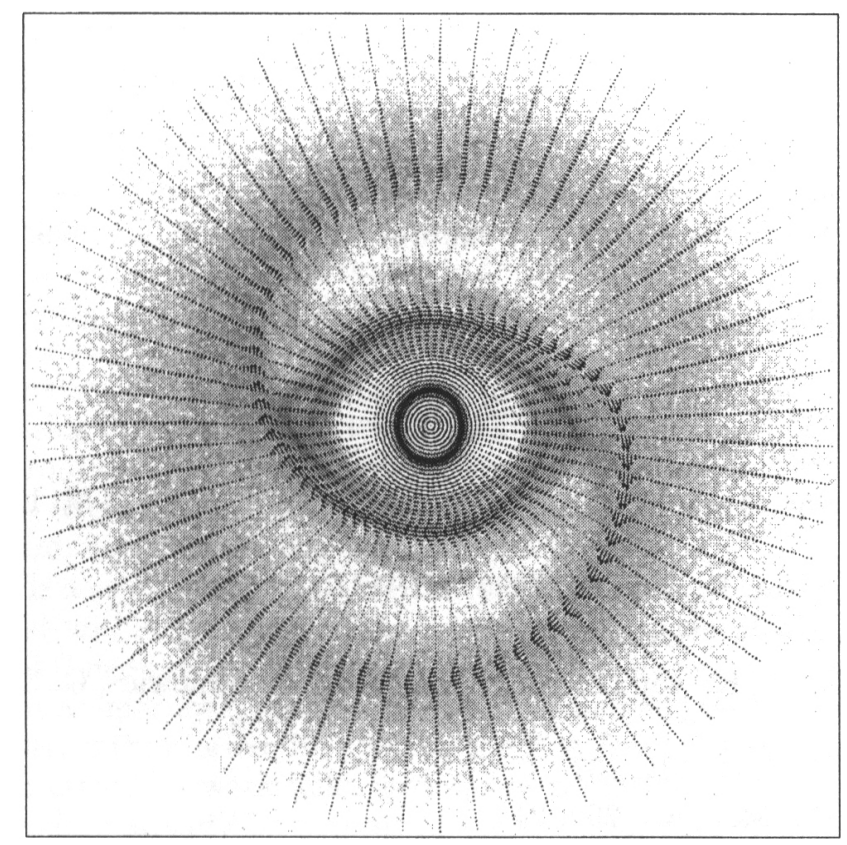

Figure 1. Magnetic field vectors and gas density (gray scale) for a model of the weakly barred galaxy IC4214 (Moss et al. 1999) 
A more challenging task is to model strongly barred systems, such as NGC1097. Preliminary attempts, again using a hydrodynamically determined velocity field (now with a shock present), do reproduce some of the significant features of the observed field (Moss et al., in preparation).

Recent detections of organized large-scale magnetic fields in slowly rotating irregular galaxies (e.g. Chyży, this Proceedings) may pose new challenges for dynamo theory, which conventionally requires significant galactic rotation for dynamo excitation.

\section{Conclusions}

Galactic dynamo theory is robust, in that it appears capable of reproducing the salient features of the large-scale fields found in a variety of disc galaxies, within a single conceptual framework. Nevertheless, substantial areas of uncertainty remain, notably the phenomenon of relatively narrow spiral arms: understanding the origin of this feature may provide a broader insight into galactic magnetism. Of course, as the sensitivity of the radio observations increases, the criteria by which the models are assessed become more severe, and it becomes necessary to use more complete physical models. An immediate objective is to model specific, rather than generic, galaxies. It is clear that this needs improved knowledge of several key quantities: noncircular velocities (including contributions not mentioned above, such as galactic fountain flows and galactic winds), the effects of spiral arms (including gas streaming, azimuthal modulation of turbulencerelated quantities, resonances between dynamo modes and pattern frequencies), and the true nature of the alpha-effect and other nonlinearities.

\section{References}

Athanassoula, L. 1992, MNRAS, 259, 345

Beck, R., Ehle, M., Shoutenkov, V., Shukurov, A., Sokoloff, D. 1999, Nat., 397, 324

Elstner, D., Otmianowska-Mazur, K., von Linden, S., Urbanik, M. 2000, A\&A, 357,129

Hanasz, M., Lesch, H. 1998, A\&A, 332, 77

Moss, D. 1998, MNRAS, 297, 860

Moss, D., Shukurov, A., Sokoloff, D.D., Beck, R., Berkhuijsen, E.M. 1998, A\&A, 335,500

Moss, D., Rautiainen, P., Salo, H. 1999, MNRAS, 303, 125

Moss, D., Shukurov, A., Sokoloff, D. 2000, A\&A, 358, 1142

Otmianowska-Mazur, K., von Linden, S., Lesch, H., Skupniewicz, G. 1997, A\&A, 323,56

Parker, E.N. 1992, ApJ, 401, 137

Shukurov, A. 1998, MNRAS, 299, 21

von Linden, S., Otmianowska-Mazur, K., Lesch, H., Skupniewicz, G. 1998, A\&A, 333,79 